Article

\title{
Fabrication of Wearable PDMS Device for Rapid Detection of Nucleic Acids via Recombinase Polymerase Amplification Operated by Human Body Heat
}

\author{
Kieu The Loan Trinh ${ }^{1}$ and Nae Yoon Lee ${ }^{2, * \mathbb{D}}$ \\ 1 Department of Industrial Environmental Engineering, Gachon University, 1342 Seongnam-daero, Sujeong-gu, \\ Seongnam-si 13120, Gyeonggi-do, Korea; tktloan@gmail.com \\ 2 Department of BioNano Technology, Gachon University, 1342 Seongnam-daero, Sujeong-gu, \\ Seongnam-si 13120, Gyeonggi-do, Korea \\ * Correspondence: nylee@gachon.ac.kr
}

check for updates

Citation: Trinh, K.T.L.; Lee, N.Y. Fabrication of Wearable PDMS Device for Rapid Detection of Nucleic Acids via Recombinase Polymerase Amplification Operated by Human Body Heat. Biosensors 2022, 12, 72. https://doi.org/10.3390/ bios12020072

Received: 5 January 2022

Accepted: 25 January 2022

Published: 27 January 2022

Publisher's Note: MDPI stays neutral with regard to jurisdictional claims in published maps and institutional affiliations.

Copyright: (C) 2022 by the authors. Licensee MDPI, Basel, Switzerland. This article is an open access article distributed under the terms and conditions of the Creative Commons Attribution (CC BY) license (https:// creativecommons.org/licenses/by/ $4.0 /)$.

\begin{abstract}
Pathogen detection by nucleic acid amplification proved its significance during the current coronavirus disease 2019 (COVID-19) pandemic. The emergence of recombinase polymerase amplification (RPA) has enabled nucleic acid amplification in limited-resource conditions owing to the low operating temperatures around the human body. In this study, we fabricated a wearable RPA microdevice using poly(dimethylsiloxane) (PDMS), which can form soft-but tight—contact with human skin without external support during the body-heat-based reaction process. In particular, the curing agent ratio of PDMS was tuned to improve the flexibility and adhesion of the device for better contact with human skin, as well as to temporally bond the microdevice without requiring further surface modification steps. For PDMS characterization, water contact angle measurements and tests for flexibility, stretchability, bond strength, comfortability, and bendability were conducted to confirm the surface properties of the different mixing ratios of PDMS. By using human body heat, the wearable RPA microdevices were successfully applied to amplify $210 \mathrm{bp}$ from Escherichia coli O157:H7 (E. coli O157:H7) and 203 bp from the DNA plasmid SARS-CoV-2 within 23 min. The limit of detection (LOD) was approximately $500 \mathrm{pg} /$ reaction for genomic DNA template (E. coli O157:H7), and $600 \mathrm{fg} /$ reaction for plasmid DNA template (SARS-CoV-2), based on gel electrophoresis. The wearable RPA microdevice could have a high impact on DNA amplification in instrument-free and resource-limited settings.
\end{abstract}

Keywords: wearable RPA microdevice; poly(dimethylsiloxane) (PDMS); human body heat; recombinase polymerase amplification (RPA)

\section{Introduction}

Nucleic acid amplification testing has become the gold standard diagnostic method applied in various fields, such as pathogen detection, early cancer diagnosis, and forensic identification [1,2]. Polymerase chain reaction (PCR) is the most common technique for amplifying nucleic acids and has been proven to be an accurate method, with high specificity and sensitivity [3-5]. Once again, during the coronavirus disease 2019 (COVID-19) pandemic, PCR has proved to be the most reliable method for the early, rapid detection of COVID-19 [6-8]. However, PCR usually requires several hours for the amplification process using a thermal cycler, which makes it difficult to utilize its applications in resourcelimited settings where the pandemic and burden of many infectious diseases are the most severe [9].

Alternative methods include isothermal amplification, such as loop-mediated isothermal amplification (LAMP) [10,11], helicase-dependent amplification (HDA) [12,13], and recombinase polymerase amplification (RPA) [14-17]. Among the various isothermal amplification methods, RPA, which was introduced in 2006, has been applied to amplify nucleic 
acids because of its high sensitivity and rapid amplification at low temperatures $\left(30-40{ }^{\circ} \mathrm{C}\right)$, which is a relatively similar temperature range to that of the human body $[18,19]$. The mild-acting temperature of RPA allows simple operation by skin contact and eliminates the need for an external heating device. Because of this benefit, RPA can be an ideal choice for building a point-of-care (POC) device for nucleic acid amplification under low-resource setting conditions. Several studies have utilized this characteristic in developing RPA devices that work at human body temperature $[20,21]$. These devices were held tightly to the skin using external supports, such as adhesive bandages or rubber bands, since tight contact with the skin is necessary for efficient heat transfer from the skin to the reaction solution.

Poly(dimethylsiloxane) (PDMS) is a silicone-based elastomer that is widely used in a variety of analytical fields owing to its flexibility, transparency, and biocompatibility [22,23]. In the field of wearable devices, PDMS is one of the most popular choices of materials because it can provide conformal contact with different surfaces and be easily wrapped around curvatures due to its low Young's modulus ( $<2 \mathrm{MPa})$ [24-26]. In addition, the assembly of PDMS devices is normally conducted by treating oxygen plasma or corona discharge to achieve permanent bonding [27]. Although this bonding method can support PDMS devices against harsh reaction conditions or distortion, an oxygen plasma machine is expensive and corona treatment over a large area is difficult. However, in applications that use mild working conditions, such as cell culturing, PDMS devices assembled without any surface treatment were able to hold tightly without any leakage [28,29]. Therefore, an RPA wearable device that works at body temperature eliminates the need for an expensive oxidation process for permanent bonding. In addition, PDMS can be tailored to improve its flexibility and adhesion for more stable contact between PDMS and PDMS and PDMS-skin by simply tuning the curing agent ratio [30].

In this study, a simple method for fabricating a wearable microdevice was introduced using flexible soft-contact PDMS to amplify nucleic acids by RPA. The PDMS was mixed with different ratios of pre-polymer and curing agent to achieve high stretchability and flexibility without changing the basic characteristics of PDMS. Water contact angle measurements and bonding performance tests with glass were performed to prove that the basic properties of PDMS remained, even with the change in curing agent ratio. Next, PDMS was used as a material for fabricating a wearable microdevice based on soft lithography and the two PDMS substrates were bonded without requiring any extra surface modification. Furthermore, a leak test was conducted to demonstrate a leak-free microdevice at room temperature. For applications, the fabricated wearable microdevice was used to amplify the DNA fragments from gDNA of a major foodborne pathogen [Escherichia coli O157:H7 (E. coli O157:H7)] and a plasmid vector of SARS-CoV-2 from COVID-19 using RPA assays.

\section{Materials and Methods}

\subsection{Materials}

TwistAmp Basic kits were purchased from TwistDx (Cambridge, UK). Poly (dimethylsiloxane) (PDMS) pre-polymer (Sylgard 184) and curing agent were purchased from Dow Corning (Midland, MI, USA). Taq DNA polymerase, PCR buffer solutions, and dNTPs were purchased from BioFact (Daejeon, Korea). Primer pairs were purchased from Bioneer (Daejeon, Korea). The DNA ladder (100 bp) was purchased from Takara (Shiga, Japan) and agarose powder was purchased from BioShop (Ontaria, Canada). Loading Star was purchased from DYNE Bio (Seongnam, Korea). SYBR Green I $(10,000 \times)$ was purchased from Lonza (Basel, Switzerland). Luria Bertani (LB) broth (with low salt) and agar powder (bacteriological) were purchased from MB cells (Seoul, Korea).

\subsection{Production of the Flexible PDMS with Different Mixing Ratios}

To produce PDMS with high flexibility as well as soft contact, mixtures with ratios of 10:1, 10:0.8, 10:0.6, and 10:0.4 (w/w, pre-polymer to curing agent) were prepared. The PDMS mixtures with different ratios were cast inside Petri dishes and cured for $2 \mathrm{~h}$ at $80^{\circ} \mathrm{C}$. To characterize each PDMS, the water contact angles, bonding performance, and 
flexibility (twisting, softness, and adhesiveness) of each sample were evaluated. A Phoenix 300 contact angle analyzer (Surface Electro Optics, Suwon-si, Gyeonggi-do, Korea) was used to measure water contact angle at the Core Facility for Bionano Materials at Gachon University. A texture analyzer (QTS 25, Brookfield, Middleboro, MA, USA) was used to evaluate the strain and bond strength. For the strain, PDMS substrates $(4 \mathrm{~cm}$ in length, $1 \mathrm{~cm}$ in width, and $600 \mu \mathrm{m}$ in thickness) were held in two grips, and the loading arm moves up at a constant speed $(100 \mathrm{~mm} / \mathrm{min})$ to achieve the "dogbone" shape of PDMS substrates. For bond strength measurement, twine was embedded into the PDMS pre-polymers and cured. After attaching two PDMS substrates, the twines were pulled apart at a speed of $100 \mathrm{~mm} / \mathrm{min}$. Each sample was measured three times, on the same day.

\subsection{Design and Fabrication of Wearable Microdevice}

Figure 1 shows the overall schematic for fabricating and operating a wearable PDMS microdevice. The wearable PDMS microdevice was fabricated using standard replica molding processes to create microchambers containing RPA reagents as shown in Figure $1 \mathrm{a}$. Briefly, the Norland optical adhesive mold (NOA mold) of the microchambers was fabricated on a PET film using a soft lithography method. Next, the pre-polymer PDMS was prepared by mixing the pre-polymer and curing reagents at 10:0.4 (w/w) ratios by weight and poured onto the NOA mold. After $2 \mathrm{~h}$ of incubation at $80^{\circ} \mathrm{C}$, the cured replica PDMS was peeled off and the inlet and outlet were made using a hole puncher $(1 \mathrm{~mm}$ in dimension). In addition, a flat PDMS thin film (1 $\mathrm{mm}$ in thickness) was fabricated using the same conditions as the replica PDMS. The patterned side of the PDMS replica was then aligned and placed in contact with the PDMS thin film to create a reversible bond due to the increased soft-contact and friction of PDMS, which did not require oxygen plasma treatment. As a result, the flexible wearable PDMS microdevice was completed, and the PDMS thin film was placed on the human skin to perform the RPA assays. In addition, Figure $1 b$ shows the schematic illustration of a wearable RPA microdevice operation process. In brief, there were two steps including RPA reagent loading and RPA reaction performed before and after adhering the wearable microdevice to the human skin. As shown in Figure 1(bi), the microdevice was covered by another thin PDMS film for enclosing the microdevice chamber after loading the RPA reagents. This avoids the sample loss while the volunteers are freely moving during the RPA reaction. After the reaction, the microdevice was removed to analyze the RPA amplicons (Figure 1(bii)). Steps of operation could be flexibly changed depending on the position of the adhered microdevice for the comfort of volunteers.

\subsection{Sample Preparation and DNA Extraction}

In this study, a foodborne pathogen (E. coli O157:H7 (ATCC 43895)) and one clinical virus (COVID-19) were tested. E. coli O157:H7 bacteria were grown in both liquid culture media and agar plates. E. coli O157:H7 was cultured in $5 \mathrm{~mL}$ of LB broth $(10 \mathrm{~g}$ of tryptone, $5 \mathrm{~g}$ of yeast extract, and $5 \mathrm{~g}$ of $\mathrm{NaCl}$ in $1 \mathrm{~L}$ of distilled water) at $37^{\circ} \mathrm{C}$ for $16 \mathrm{~h}$, with constant shaking at $200 \mathrm{rpm}$. Viable counts were determined by performing serial dilution plating on solid LB agar media for E. coli O157:H7 and incubating at $37^{\circ} \mathrm{C}$ for $16 \mathrm{~h}$. For DNA extraction from E. coli O157:H7, the bacterial DNA was extracted from a solution that was cultured overnight via the Wizard Genomic DNA purification kit. The extracted gDNA was stored at $4{ }^{\circ} \mathrm{C}$ for short-term storage. In addition, with the ongoing global pandemic of COVID-19, a commercially available SARS-CoV-2 plasmid DNA was employed as a template to amplify the DNA for potential clinical applications. 
(a) Microdevice fabrication process

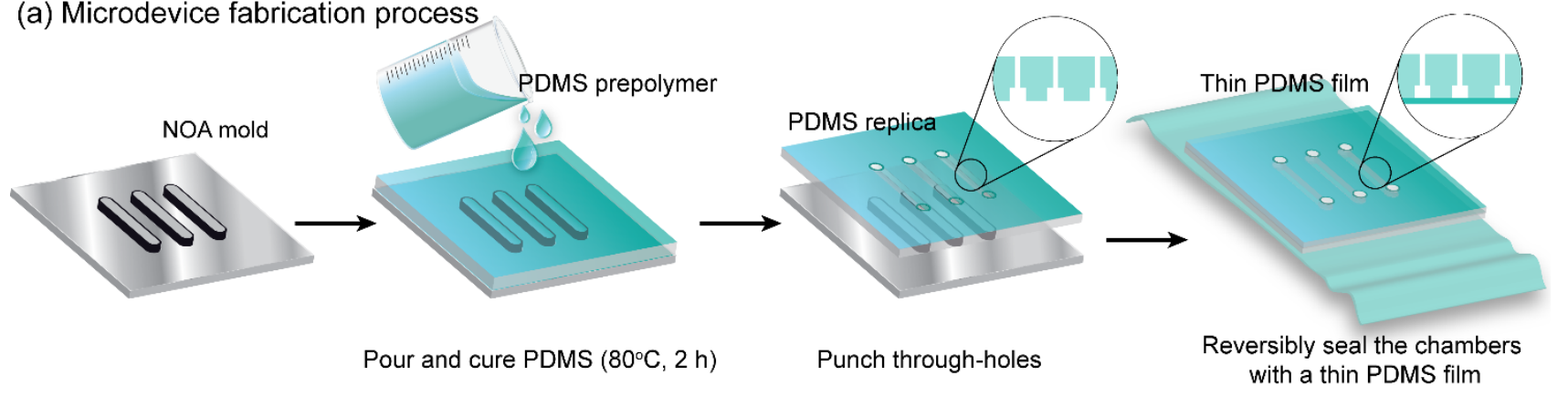

(b) Wearable RPA operation process

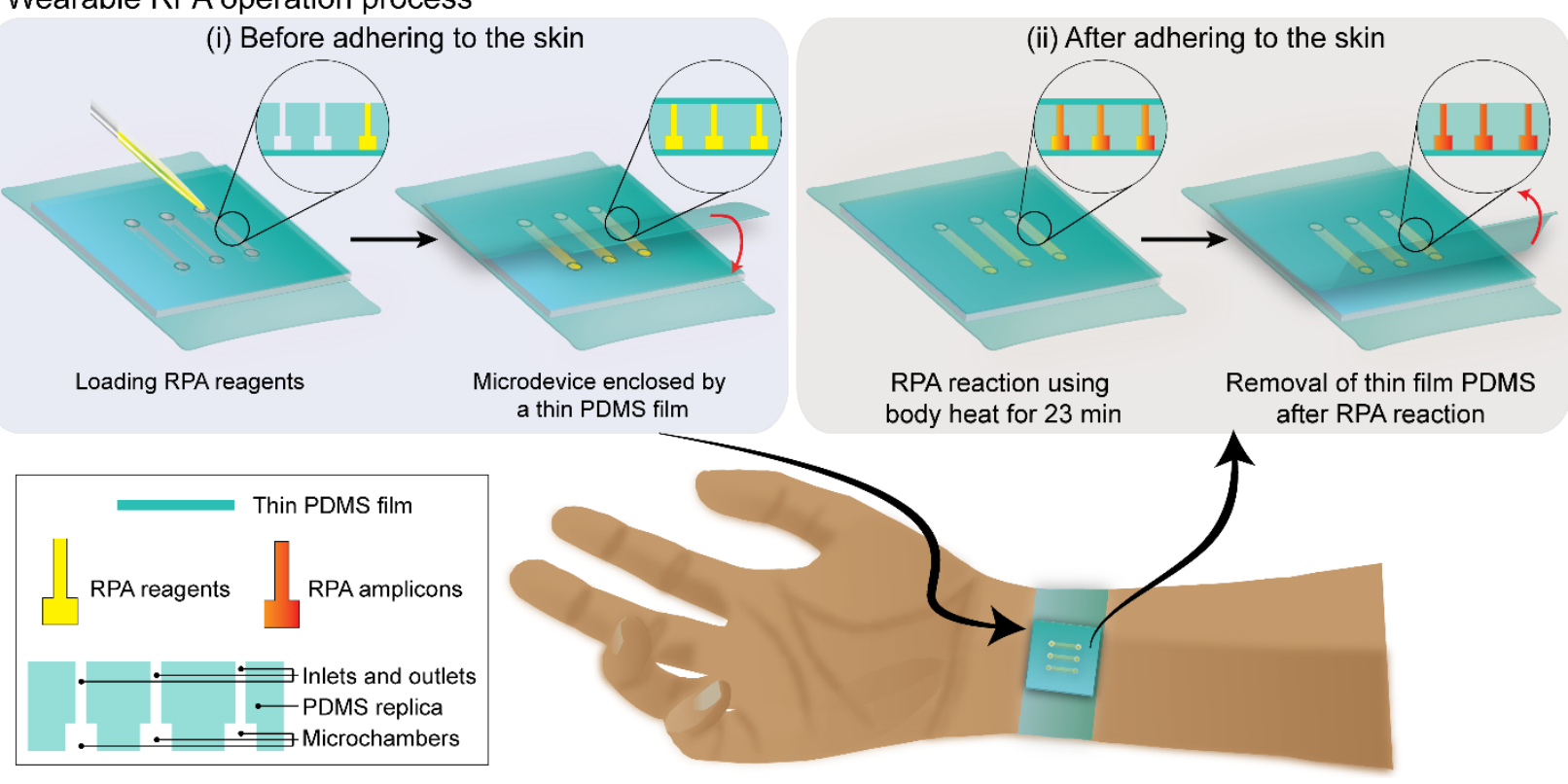

Figure 1. (a) Schematic illustration showing the fabrication of a wearable PDMS microdevice. (b) Schematic illustration showing the operation of a wearable PDMS microdevice.

\subsection{Temperature Measurement}

In this study, human body heat was used to conduct all of the RPA assays. Human body heat was measured using a non-contact precision infrared thermometer (Daihan Scientific Co., Ansung-si, Gyeonggi, Korea). In the comparison, an IR camera was used to measure the human body heat as a control experiment. In this way, $1 \mathrm{~mm}$ thin PDMS substrates were attached to the human body at four positions, as described previously. To evaluate the temperature distribution of human skin, 10 spots were randomly selected from each position of the chest, forearm, wrist, and fist for a 30 min period.

\subsection{Nucleic Acid Amplification by RPA}

The primer sequences used to amplify $210 \mathrm{bp}$ of the Shiga toxin gene in E. coli O157:H7 were designed as follows: 5'-TGT AAC TGG AAA GGT GGA GTA TAC A-3' (forward) and 5'-GCT ATT CTG AGT CAA CGA AAA ATA AC-3' (reverse) [31]. The primer sequences for amplifying a 203 bp gene fragment in SARS-CoV-2 plasmid DNA were as follows: 5'-ACT CTT TCG TGA GCG AGG AA-3' (forward) and 5'-CCT GGA GCT GTT CAG GTT CT-3' (reverse). A SARS-CoV-2 plasmid DNA (100 ng/ $\mu \mathrm{L})$ was further diluted and added to the RPA reagent to obtain a final template concentration of $600 \mathrm{fg} /$ reaction. The extracted DNA of E. coli O157:H7 $(240 \mathrm{ng} / \mu \mathrm{L})$ was further diluted and added to the RPA reagent to achieve a final template concentration of $500 \mathrm{pg} /$ reaction. To perform RPA, on-tube RPA was demonstrated and optimized according to the instructions provided in the TwistAmp Basic kits. The detailed schematics for RPA for DNA amplification were illustrated as shown in Figure S1. Briefly, the RPAs were performed using a $15 \mu \mathrm{L}$ sample 
containing a $2 \times$ reaction buffer, $0.64 \mathrm{mM}$ dNTP mixture, $10 \times$ Basic E-mix, and $10 \mu \mathrm{M}$ of forward and reverse primers. All RPA reactions were incubated for 23 min using human body heat for all experiments. For control experiments, conventional PCR assays were performed on a thermal cycler (Bio-Rad C1000 thermal cycler) to compare the sensitivity and specificity of the RPA reactions. The PCRs were performed using a $25 \mu \mathrm{L}$ sample containing a $5 \mu \mathrm{L}$ buffer, $0.16 \mathrm{nM}$ dNTPs mixture, $0.5 \mu \mathrm{M}$ forward and reverse primers and $0.5 \mathrm{U} / \mu \mathrm{L}$ of Taq DNA polymerase. The input DNA of SARS-CoV-2 plasmid DNA and extracted DNA of E. coli O157:H7 were prepared at the same concentration in the RPA assays. The PCR amplifications were performed for 32 thermal cycles at $95^{\circ} \mathrm{C}$ for $30 \mathrm{~s}$ for denaturation, $54{ }^{\circ} \mathrm{C}$ for $30 \mathrm{~s}$ for annealing, and $72{ }^{\circ} \mathrm{C}$ for $30 \mathrm{~s}$ for elongation, with an initial denaturation at $95^{\circ} \mathrm{C}$ for $3 \mathrm{~min}$, followed by a final extension step at $72{ }^{\circ} \mathrm{C}$ for $5 \mathrm{~min}$. The amplicons were confirmed via gel electrophoresis on a $1.5 \%$ agarose gel. For rapid visual amplification, $0.1 \mu \mathrm{L}$ of SYBR Green I $(10 \times)$ was added to the RPA samples to realize the on-site detection of amplicons using an inverted fluorescence microscope (Olympus IX71) and the fluorescence signals were recorded and analyzed using ProgRes Capture Pro 2.8 software (Jenoptik). The intensities of RPA and PCR target bands from the gel images were further evaluated using ImageJ software.

\section{Results}

\subsection{Water Contact Angle Measurement}

As shown in Figure 2, contact angle measurements were performed to evaluate the effect of the mixing ratio of the PDMS pre-polymer and curing agent on the PDMS surface properties. The measured contact angles of PDMS with different mixing ratios (10:1, 10:0.8, 10:0.6, and 10:0.4 $(w / w)$ ) were approximately $112.7 \pm 1.5^{\circ}, 112.9 \pm 0.5^{\circ}, 114.7 \pm 1.0^{\circ}$, and $115.8 \pm 0.7^{\circ}$, respectively. As shown in Figure $2 b$, after the oxygen plasma treatment, all contact angles dropped to less than $10^{\circ}$, indicating the hydrophilic stage of the PDMS surfaces. Based on the results shown in Figure $2 a, b$, we can conclude that the innate hydrophobicity of PDMS was not altered due to the reduced level of the curing agent.

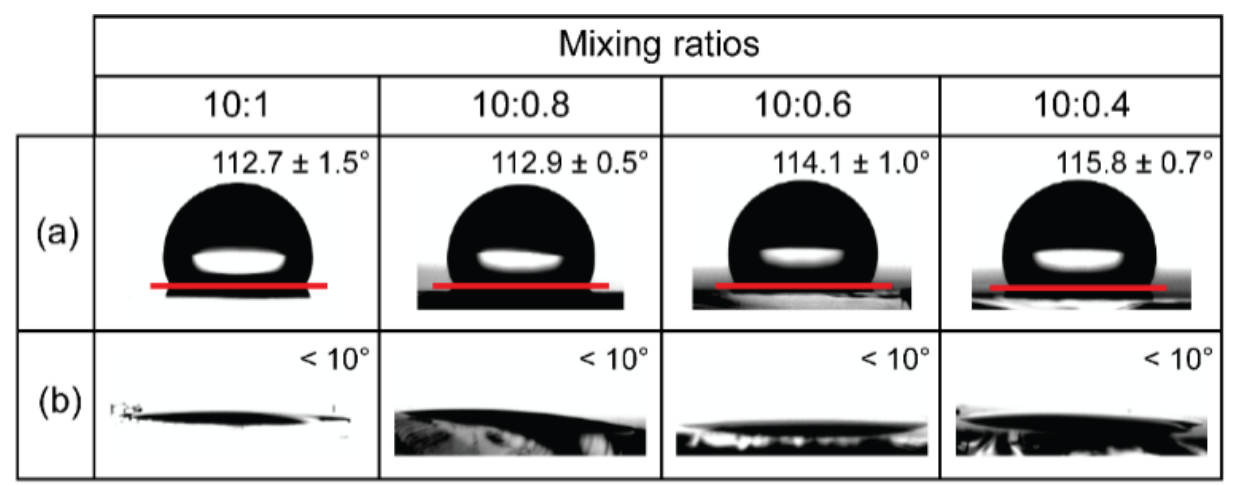

Figure 2. Characterization of PDMS substrates with different mixing ratios. Results of water contact angles measurements on PDMS substrates: (a) Pristine PDMS. (b) Oxygen plasma treatment of the PDMS. Five measurements were taken and averaged.

\subsection{Characterization of the PDMS Substrates with Different Mixing Ratios}

To utilize PDMS for wearable device fabrication, a series of key performance indicators, including flexibility, stretchability, bond strength, comfortability, and bendability, were measured and characterized. First, a flexibility test was conducted to demonstrate that PDMS can be twisted, displaying flexible properties [32]. As shown in Figure 3a, the $2 \mathrm{~mm}$ thick PDMS with mixing ratios of 10:1, 10:0.8, 10:0.6, and 10:0.4 (w/w) allowed for twisting at $360^{\circ}, 540^{\circ}, 720^{\circ}$, and $1260^{\circ}$, respectively, and did not deform the PDMS. As shown in Figure 3b, $600 \mu \mathrm{m}$ thick PDMS with a mixing ratio of 10:1, 10:0.8, 10:0.6, and 10:0.4 $(w / w)$ were gradually pulled until the maximum stretch. The 10:1 PDMS was only able to stretch slightly; however, with decreasing curing agent ratio, the strain percentage 
significantly increased to values of $126 \pm 3.8 \%, 157 \pm 1.4 \%, 203 \pm 3.0 \%$, and $320 \pm 3.0 \%$ for 10:1, 10:0.8, 10:0.6, and 10:0.4 (w/w), respectively. The inset shows photographs of the PDMS substrate with a 10:0.4 $(w / w)$ mixing ratio before and after stretching. These results show that the 10:0.4 PDMS substrate is highly suitable for wearable devices owing to its highly flexible and stretchable nature. As shown in Figure $3 c$, the bond strengths of the PDMS-PDMS bonding with mixing ratios of 10:1, 10:0.8, 10:0.6, and 10:0.4 $(w / w)$ were measured to be approximately $6.5 \pm 0.2 \mathrm{kPa}, 7.0 \pm 0.1 \mathrm{kPa}, 7.4 \pm 0.5 \mathrm{kPa}$, and $9.5 \pm 0.2 \mathrm{kPa}$, respectively. It is worth noting that two PDMSs adhered to each other without requiring surface modification. As a result, the 10:0.4 (w/w) mixing ratio of PDMS achieved the highest bond strength among the other mixing ratios. In addition, the 10:0.4 mixing ratio of PDMS was further employed for comfortability, bendability, and leak tests, as shown in Figure S2. As shown in Figure S2, the PDMS mixing ratio of 10:0.4 (w/w) also demonstrates a high softness and adhesiveness due to its high friction with human skin or other materials, as per previous studies [26]. The wearable microdevice was firmly attached to the human forearm without external support and the contact between the wearable microdevice and human skin was maintained, even when the device was turned upside down, as shown in Figure S2a. Therefore, the microdevice could withstand the random movement of the wearers. Furthermore, Figure S2c shows the results of the static leak test performed on the flexible wearable microdevice. Although non-plasma bonding of PDMS was applied to fabricate the wearable microdevice, it could still prevent the ink solution from leaking for over $96 \mathrm{~h}$ at room temperature. The results demonstrate that the lower curing agent ratio results in excellent flexibility for using PDMS in various wearable microdevices-related applications. For all subsequent experiments, a 10:0.4 $(w / w)$ mixing ratio of PDMS was used owing to its incredible flexibility, which satisfies the requirements of a wearable RPA microdevice.

\subsection{Sensitivity Analysis of the RPA Assays Using Human Body Heat for the Detection of E. coli O157:H7}

The sensitivity test is one of the most important criteria for the early detection of bacterial pathogens based on DNA amplification. Therefore, the sensitivity of RPA was evaluated using tenfold serial dilutions of the extracted gDNA from E. coli O157:H7 bacteria, as shown in Figure 4a. As shown in the gel image, lanes 1-8 represent from 50 fg to $50 \mathrm{ng} /$ reaction. From the results, RPA successfully amplified the $210 \mathrm{bp}$ target gene from genomic DNA when the input DNA concentration was from $500 \mathrm{pg}$ to $50 \mathrm{ng} /$ reaction. Accordingly, the limit of detection (LOD) of RPA was $500 \mathrm{pg} /$ reaction within $23 \mathrm{~min}$ of incubation using human body heat. In addition, by using the same amount of input genomic DNA, the LOD of PCR was also conducted to compare with the LOD of RPA assays, as shown in Figure 4b. Similarly, the results indicated that PCR and RPA shared the same sensitivity value (500 pg/reaction) based on gel electrophoresis. However, in the case of PCR, the target band intensity signal was relatively weak when the input DNA was $500 \mathrm{pg} /$ reaction, compared with RPA results. It can be concluded that the LOD of RPA can achieve a similar sensitivity for the same amount of input DNA as PCR while having the advantages of easier access and operation for the amplification reaction using point-of-care testings (POCTs). Furthermore, $500 \mathrm{pg}$ (genomic DNA) was considered as a limit of detection for detecting E. coli O157:H7 using both RPA and PCR assays, and this concentration value was used for all subsequent on-tube and on-chip RPA experiments. 

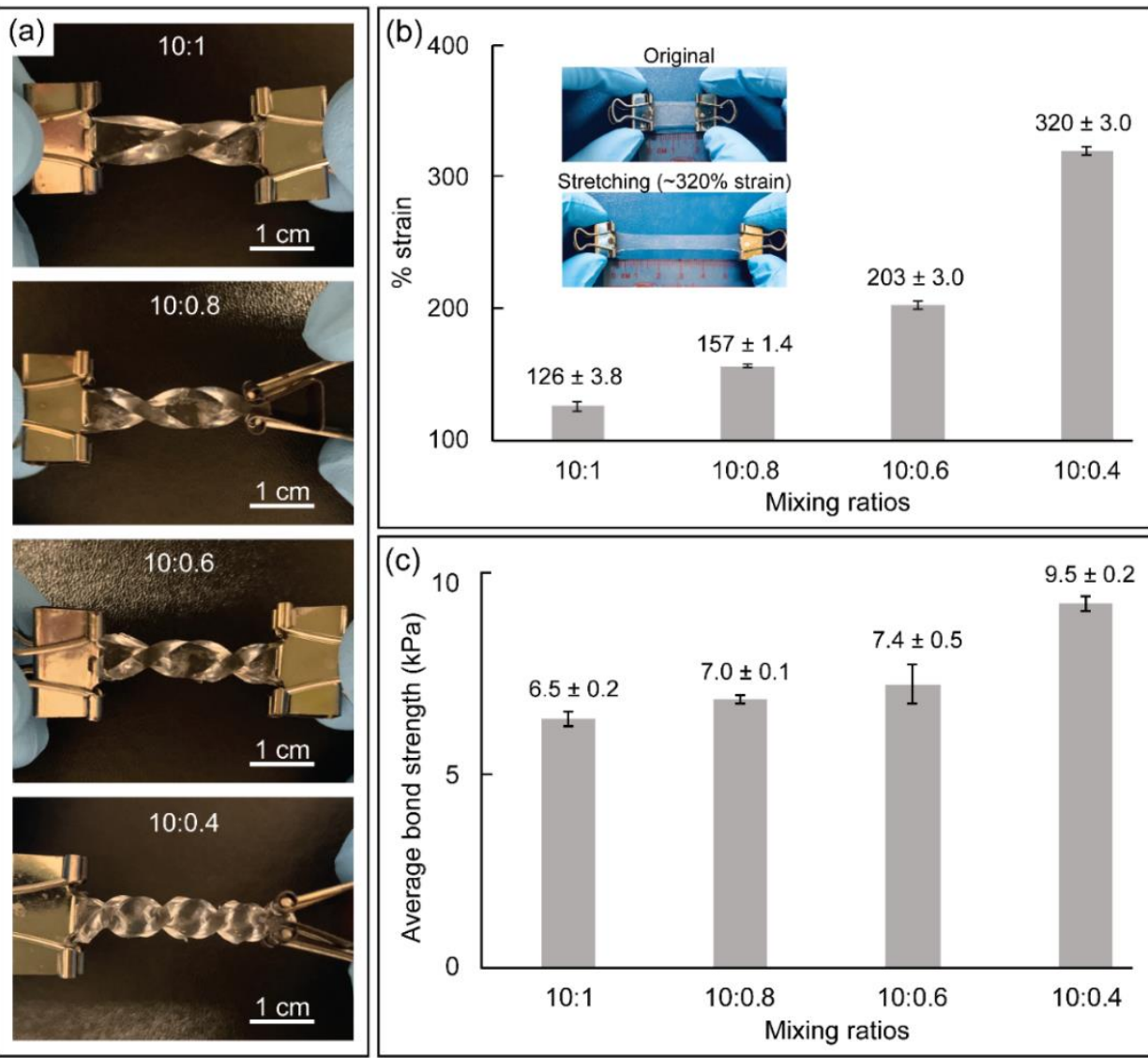

Figure 3. (a) Twisting test showing the flexibility of the PDMS fabricated with different mixing ratios of the pre-polymer and a curing agent. (b) Strain resolution of the PDMS substrate with different mixing ratios. The inset shows the photographs of the 10:0.4 (w/w) mixing ratio of PDMS substrate at different strains (original and stretching conditions). (c) Results of bond strength performed on PDMS-PDMS with different mixing ratios. All experiments were performed in triplicate.

\subsection{Results of on-Chip RPA Performed Using Human Body Heat for the Detection of E. coli O157:H7}

Figure 5 shows the RPA results from using human body heat to amplify E. coli O157:H7. The soft-contact wearable PDMS microdevices formed tight contact with human skin at most positions, such as the chest (P1), forearm (P2), wrist (P3), and fist (P4), as shown in Figure 5a. It was clearly shown that the wearable PDMS microdevice directly adhered to the skin without the use of any support bandages or tapes because of its soft-contact and flexibility. From this result, the wearable PDMS microdevice can maintain a conformal contact with the skin during the RPA reaction, reducing heat loss and improving heat transfer to achieve a homogenous heat profile. Before performing RPA using human body heat, RPAs were first performed on thermocycler with a temperature ranging from $30-40{ }^{\circ} \mathrm{C}$ as shown in Figure S3a. Afterward, RPA was performed on three volunteers with slightly different body temperatures (Figure S3b). Interestingly, all RPAs were successfully performed with negligible difference in the target intensities. From these results, we can conclude that the RPA can take place under normal body heat even with slight variations from human to human. Although the leak test result strongly supports the robustness of the sealing between the two pieces of PDMS over $96 \mathrm{~h}$ at room temperature (Figure S2c), it is worth noting that the wearable RPA device is sealed by reversible bonding. Therefore, it would be better to minimize movements such as forceful pushing or pulling during the RPA amplification to avoid any chances of leakage, which may cause cross-contamination or a false-positive result. Moreover, for high-risk DNA samples, the wearable RPA microdevice can be irreversibly sealed using the oxygen plasma bonding method; it would be a more 
preferred and safer approach for practical application. To demonstrate the applicability of this device, RPAs were conducted by amplifying the $210 \mathrm{bp}$ target gene from E. coli O157:H7 using human body heat at four positions, as mentioned previously. Before performing on-chip RPA amplification, on-tube RPAs were first performed using a $0.5 \mathrm{~mL}$ tube to evaluate the effect on the amplification efficiency of human body heat at different positions, as shown in Figure 5b. The tube was attached to the human skin for RPA via a commercially available adhesive tape, as shown in Figure S4. As shown in the gel image, RPAs successfully amplified the $210 \mathrm{bp}$ target gene using human body heat from the P1 to P4 positions (lanes $2-5$ ). The target band was clearly expressed as a single band, indicating the specificity of the amplification, whereas no band was observed in the negative control (lane 1). The intensities of the RPA products obtained from P2 and P3 positions were approximately $53.2 \%$ of those obtained from $\mathrm{P} 1$ and $\mathrm{P} 4$, still demonstrating distinguishable amplicons, probably due to its higher heat distribution and stable temperature during RPA assays, as shown in Figures S5 and S6. The RPA using body heat was further tested on the microdevice, as shown in Figure $5 \mathrm{c}$. The $210 \mathrm{bp}$ target band was successfully amplified in the chambers within $23 \mathrm{~min}$ of incubation at the four positions (P1-P4). Once again, P1 and P4 (lanes 1 and 4) exhibited higher and clearer amplification results of the target band than those obtained from P2 and P3 (lanes 2 and 3), which corresponded with the on-tube results, as shown in Figure $5 \mathrm{~b}$. The intensities were approximately 2 -fold higher (57.9\%) for the results obtained from P1 and P4 compared to those obtained from P2 and P3. Therefore, the on-chip RPA results showed an almost similar trend to those obtained from the on-tube RPA. From these results, the fabricated wearable microdevice can be applied for DNA amplification through RPA assays using human body heat from various positions. Among the four positions, the forearm was selected to perform all RPA assays for easier access and a comfortable operating position.

\subsection{Amplification of Plasmid DNA of COVID-19 Using the Wearable RPA Device}

In addition to foodborne pathogens, the wearable RPA microdevice was also applied to amplify the $203 \mathrm{bp}$ gene fragment from the plasmid DNA of SARS-CoV-2. Before performing on-chip RPA, the LOD of RPA was determined to be approximately $600 \mathrm{fg} /$ reaction, as shown in Figure S7a. Based on this result, $600 \mathrm{fg}$ were added into RPA reagents for all the following RPA assays. As shown in Figure 6, after 23 min of incubation, the $203 \mathrm{bp}$ target gene was successfully amplified on the tube (Figure 6a) and wearable microdevice (Figure $6 \mathrm{~b}$ ), as confirmed by gel electrophoresis. In both gel images, lane 1 shows a negative control without the target band. The target band was displayed on a gel image with high specificity and selectivity (lanes 2 and 3 for on-tube RPA; lane 2 for on-chip RPA). In addition, PCR was also utilized to confirm the specificity and sensitivity of RPAs for amplifying the $203 \mathrm{bp}$ target gene by using the same amount of input DNA, and the LOD was approximately $60 \mathrm{fg} /$ reaction (Figure S7b), which was slightly higher in sensitivity as compared with the results obtained from RPA assays. Based on these results, we concluded that the wearable soft-contact RPA microdevice could be energetically applied for rapid detection of SARS-CoV-2 in a simpler manner and low-resource settings. 
(a) RPA
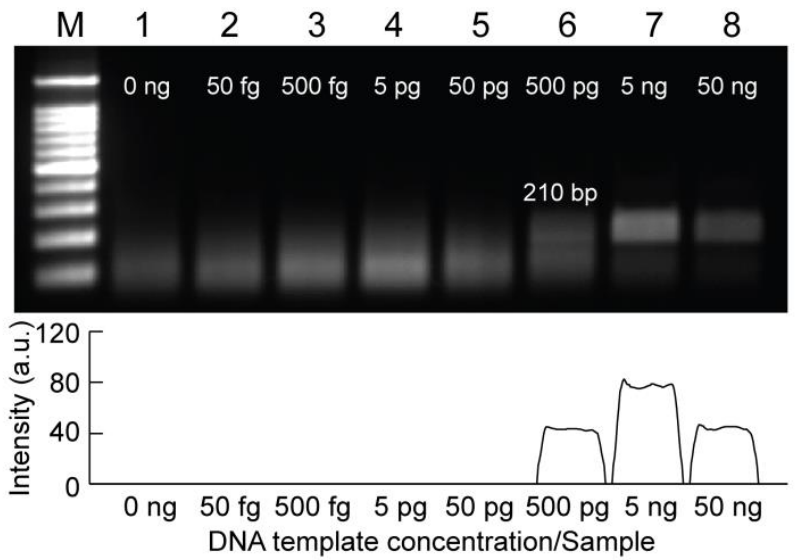

(b) PCR
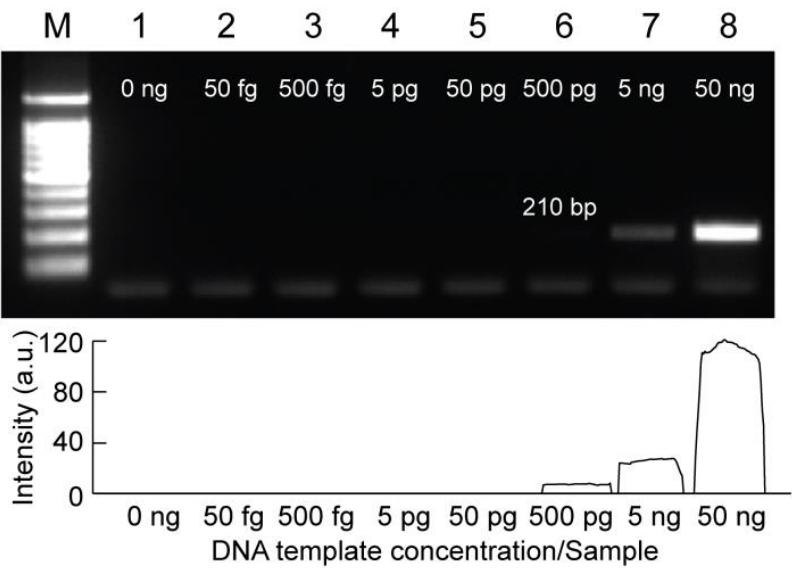

Figure 4. Results of gel electrophoresis showing the sensitivity using tenfold serial dilutions of gDNA input from E. coli O157:H7 bacteria. (a) On-tube RPA results. (b) On-tube PCR results. Lane M is the 100 bp DNA ladder. Lanes 1-8 show the amplification results when input gDNA concentrations were $50 \mathrm{fg}$ to $50 \mathrm{ng} / \mathrm{sample}$, respectively. Relative intensity scales of the target amplicons were shown below each gel image. All the experiments were repeated three times.

\subsection{On-Site Detection of Amplicons Using SYBR Green I-Based Fluorescence Signal}

In addition, to shorten the detection time of amplicons, SYBR Green I was added to the RPA sample after 23 min of incubation for the rapid detection-based fluorescence method. It is worth noting that SYBR Green I was only added after the microdevice was separated from the human body owing to a safety concern. Fluorescence images of two zones of the microchambers were obtained from the fluorescence images of two target genes (203 bp from plasmid SARS-CoV-2 and $210 \mathrm{bp}$ from E. coli O157:H7), as shown in Figure 7a. The RPA results successfully amplified two gene fragments using a wearable PDMS microdevice, as shown in the gel image (Figure S8). Figure $7 b, c$ show the fluorescence images of onchip amplification of the $203 \mathrm{bp}$ target band (plasmid SARS-CoV-2) and $210 \mathrm{bp}$ target band (E. coli O157:H7) with SYBR Green I added after RPA assays. Based on these results, the strong green fluorescence signals of SYBR Green I intercalated with RPA amplicons (positive sample) of the on-chamber amplifications were significantly different from those of the negative sample, indicating consistent results between fluorescence measurements and gel electrophoresis, as shown in Figure S8. It is also noticed that the RPA reagents may contain some components that can strongly react with SYBR Green I, resulting in some high-intensity dots in the fluorescence images, as shown in Figure 7b,c. Thus, the successful on-site detection of RPA amplicons via fluorescence signals demonstrates the application potential of the wearable PDMS microdevice in resource-limited settings with high DNA amplification efficiency and rapid detection of amplicons. 


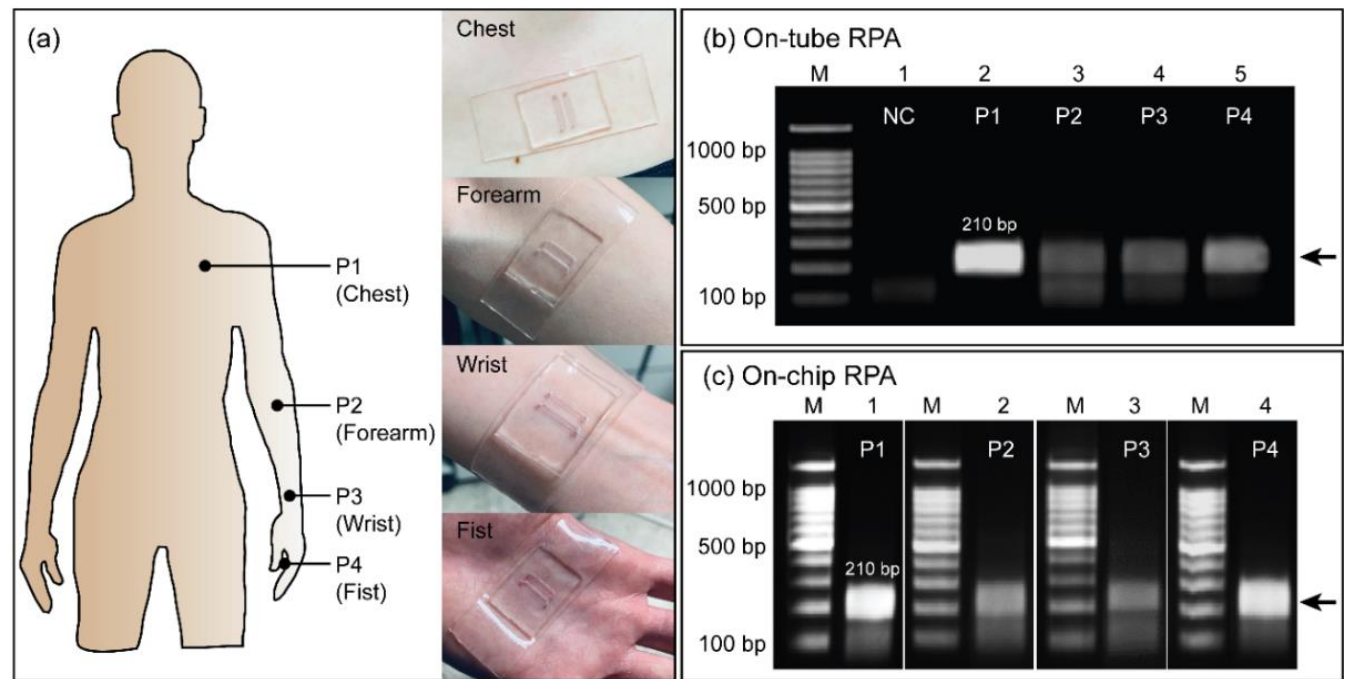

Figure 5. Wearable PDMS microdevice for RPA assays was performed using human body heat. (a) Illustration of four different positions (P1—chest; P2-forearm; P3-wrist; and P4-fist) and real photographs of the wearable PDMS microdevice adhered on a volunteer's skin which was utilized to perform RPA using human body heat. (b) Agarose gel electrophoresis of tube-based RPA assays using human body heat at four different positions (P1 to P4). (c) Agarose gel electrophoresis of the wearable PDMS microdevice-based RPA assays using human body heat at four different positions (P1 to P4).

(a) On-tube RPA

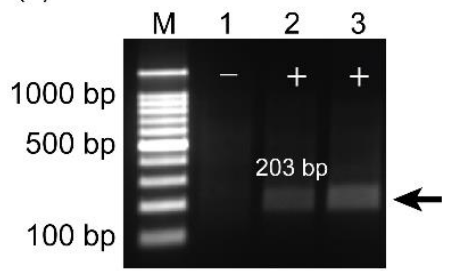

(b) On-chip RPA

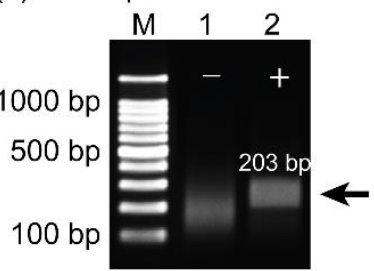

Figure 6. (a) On-tube RPA results of a 203 bp target gene using plasmid DNA of SARS-CoV-2. (b) Onchip RPA results of a 203 bp target gene from plasmid DNA of SARS-CoV-2. Lane M is the $100 \mathrm{bp}$ DNA ladder. Lane 1 is a negative sample. Lanes 2 and 3 are the positive samples. All the experiments were repeated three times.
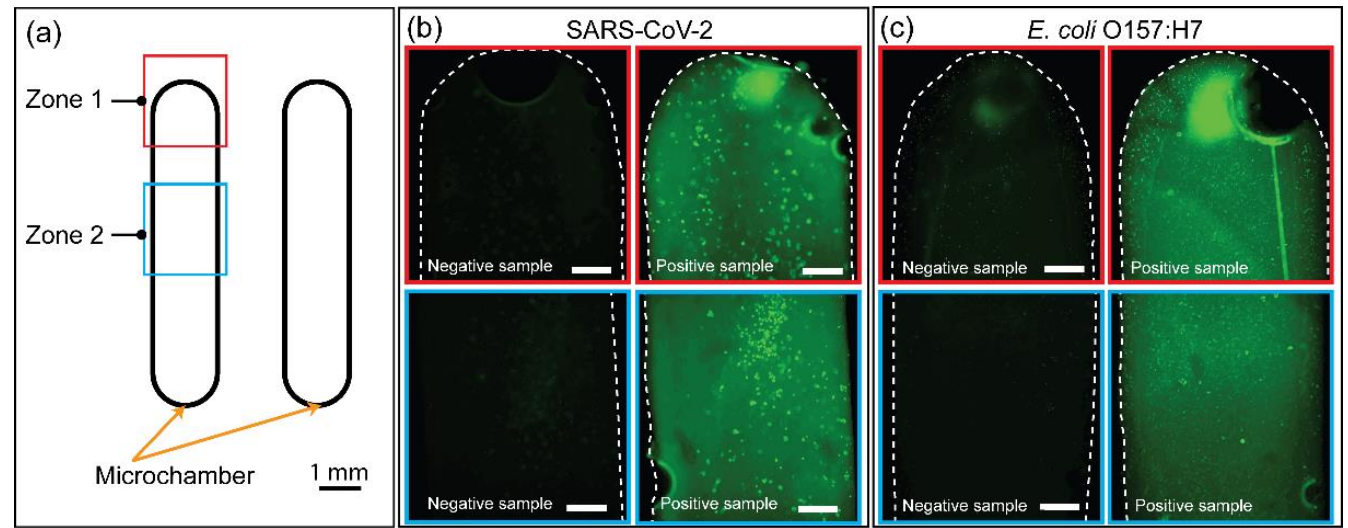

Figure 7. On-site detection of amplicons using the wearable RPA device. (a) Schematic illustration of the microdevice indicating two zones where the fluorescence images were captured. (b) Fluorescence signals of amplicons from DNA plasmid SARS-CoV-2 inside the PDMS microdevice. (c) Fluorescence signals of amplicons from E. coli O157:H7 inside the PDMS microdevice. Scale bars in $(\mathbf{b}, \mathbf{c})$ represent $200 \mu \mathrm{m}$. 


\section{Conclusions}

In summary, we fabricated a wearable RPA microdevice utilizing PDMS substrates that can perform DNA amplification via human body heat. To produce soft-contact PDMS, the PDMS substrate was developed with high flexibility and adhesion by reducing the amount of curing agent in the mixing ratio of PDMS, without changing the PDMS properties. As a result, the mixing ratio of the 10:0.4 (w/w) PDMS substrate not only improved contact between the wearable microdevice and human skin but was also able to realize PDMSPDMS bonding without surface modification. As compared with other wearable RPA devices which use additional support for holding the device such as a wristband, the current device offers wide ranges in size forming highly stable and conformal contact on human skin. Thus, the wearable microdevice was successfully employed to amplify two target genes for the detection of E. coli O157:H7 from gDNA and COVID-19 from DNA plasmid SARS-CoV-2 using human body heat with high sensitivity and specificity. By incorporating a sample preparation step, the current platform can be more suitable for POCT. In addition, the sensitivity of the current platform can be further improved by being integrated with smartphone-based detection, making the device more affordable and accessible in resource-limited settings.

Supplementary Materials: The following supporting information can be downloaded at: https: //www.mdpi.com/article/10.3390/bios12020072/s1, Figure S1: (a) The key ingredients of RPA assays include DNA template, primers, nucleotides, recombinase, DNA polymerase, and singlestrand DNA binding protein. (b) DNA amplification using the RPA technique. (i) Recombinaseprimer complexes scan DNA for complementary sequences. Recombinase inserts primers via strand exchange. Single-strand DNA binding protein (SSB) binds to displaced strands, stabilizing the bound primer. (ii,iii) The primer was extended to the new strand by DNA polymerase. The parental strand is displaced, and elongation continues. (iv) Two copies of DNA are formed. The drawings were modified from [33]; Figure S2: (a) Photographs showing the wearable PDMS microdevice attached on the forearm. (b) Photographs showing 10:0.4 (w/w) mixing ratio of PDMS and prepolymer demonstrating highly skin-compatible mechanical and soft-contact performance. (c) Results showing the leak test observed over 96 h; Figure S3: The $210 \mathrm{bp}$ target gene from E. coli O157:H7 was successfully amplified based on RPA technique using thermal cycler with temperature ranging from 40 to $30^{\circ} \mathrm{C}$. Lane 1 is a negative control. Lanes 2-7 are RPA products obtained from reactions performed from 40 to $30{ }^{\circ} \mathrm{C}$ at $2{ }^{\circ} \mathrm{C}$ intervals. (b) The $210 \mathrm{bp}$ target gene from E. coli O157:H7 was successfully amplified using RPA technique and human body heat with three different volunteers. Lane 1 is a negative control. Lanes 2-4 are RPA products obtained from three different volunteers. Lane M is 100 bp ladder; Figure S4: Illustration showing four positions for attaching tubes for RPA (P1-chest; P2 - forearm; P3 - wrist; and P4-fist) and real photographs of the tubes for RPA adhered on a volunteer's skin at four designated positions using tape; Figure S5: Temperature variations measured on the wearable RPA microdevice at four positions, P1 - chest, P2 - forearm, P3 - wrist, and $\mathrm{P} 4$ - fist. Each temperature was measured ten times using a non-contact precision infrared thermometer; Figure S6: Temperature measurement. (a) Graphs showing the temperature variations measured on the wearable RPA microdevice at four positions, P1—chest, P2-forearm, P3-wrist, and P4-fist using an IR camera. (b) IR camera images showing the temperatures measured at P3-wrist and P4-fist for $60 \mathrm{~min}$. Each temperature was measure ten times and averaged; Figure S7: Results of agarose gel electrophoresis showing the sensitivity using tenfold serial dilutions of plasmid DNA from SARS-CoV-2. (a) On-tube RPA results. (b) On-tube PCR results. Lane M is the 100 bp DNA ladder. Lanes 1-8 show the amplification results when input plasmid DNA concentrations were $0.06 \mathrm{fg}$ to $60 \mathrm{pg} / \mathrm{sample}$, respectively. All the experiments were repeated three times.; Figure S8: Agarose gel electrophoresis images of $203 \mathrm{bp}$ (SARS-CoV-2) and $210 \mathrm{bp}$ (E. coli O157:H7) target genes obtained using the wearable PDMS microdevice and human body heat. Lane M is the $100 \mathrm{bp}$ DNA ladder. Lanes 1 and 2 are the negative and positive samples of SARS-CoV-2, respectively. Lanes 3 and 4 are the negative and positive samples of $E$. coli $\mathrm{O} 157: \mathrm{H7}$, respectively.

Author Contributions: Conceptualization, K.T.L.T. and N.Y.L.; methodology, K.T.L.T. and N.Y.L.; software, K.T.L.T.; validation, K.T.L.T.; formal analysis, K.T.L.T.; investigation, K.T.L.T.; resources, N.Y.L.; data curation, N.Y.L.; writing—original draft preparation, K.T.L.T.; writing—review and 
editing, K.T.L.T. and N.Y.L.; supervision, N.Y.L.; project administration, N.Y.L.; funding acquisition, N.Y.L. All authors have read and agreed to the published version of the manuscript.

Funding: This work was supported by the National Research Foundation of Korea (NRF) grant funded by the Korea government (MSIT) (No. NRF-2020R1A2B5B01001971) and also by Basic Science Research Program through the National Research Foundation of Korea (NRF) funded by the Ministry of Education (2021R1A6A1A03038996).

Institutional Review Board Statement: Not applicable.

Informed Consent Statement: Informed consent was obtained from all subjects involved in the study.

Data Availability Statement: The data presented in this study are available on request from the corresponding author.

Conflicts of Interest: The authors declare no conflict of interest.

\section{References}

1. Louie, M.; Louie, L.; Simor, A.E. The role of DNA amplification technology in the diagnosis of infectious diseases. CMAJ 2000, 163, 301-309. [PubMed]

2. LaBarre, P.; Boyle, D.; Hawkins, K.; Weigl, B. Instrument-free nucleic acid amplification assays for global health settings. Proc. SPIE Int. Soc. Opt. Eng. 2011, 16, 802920.

3. Ahrberg, C.D.; Manz, A.; Chung, B.G. Polymerase chain reaction in microfluidic devices. Lab Chip 2016, 16, 3866-3884. [CrossRef] [PubMed]

4. Kwon, N.Y.; Ahn, J.J.; Kim, J.-H.; Kim, S.Y.; Lee, J.H.; Kwon, J.-H.; Song, C.-S.; Hwang, S.Y. Rapid subtyping and pathotyping of avian influenza virus using chip-based RT-PCR. BioChip J. 2019, 13, 333-340. [CrossRef]

5. Trinh, K.T.L.; Stabler, R.A.; Lee, N.Y. Fabrication of a foldable all-in-one point-of-care molecular diagnostic microdevice for the facile identification of multiple pathogens. Sens. Actuators B Chem. 2020, 314, 128057. [CrossRef]

6. Tahamtan, A.; Ardebili, A. Real-time RT-PCR in COVID-19 detection: Issues affecting the results. Expert. Rev. Mol. Diagn. 2020, 20, 453-454. [CrossRef] [PubMed]

7. Shen, M.; Zhou, Y.; Ye, J.; AL-maskri, A.A.A.; Kang, Y.; Zeng, S.; Cai, S. Recent advances and perspectives of nucleic acid detection for coronavirus. J. Pharm. Anal. 2020, 10, 97-101. [CrossRef]

8. Teymouri, M.; Mollazadeh, S.; Mortazavi, H.; Ghale-noie, Z.N.; Keyvani, V.; Aghababaei, F.; Hamblin, M.R.; AbbeszadehGoudarzi, G.; Pourghadamyari, H.; Hashemian, S.M.R.; et al. Recent advances and challenges of RT-PCR tests for the diagnosis of COVID-19. Pathol. Res. Pract. 2021, 221, 153443. [CrossRef]

9. Gorgannezhad, L.; Stratton, H.; Nguyen, N.-T. Microfluidic-based nucleic acid amplification systems in microbiology. Micromachines 2019, 10, 408. [CrossRef]

10. Trinh, T.N.D.; Lee, N.Y. A rapid and eco-friendly isothermal amplification microdevice for multiplex detection of foodborne pathogens. Lab Chip 2018, 18, 2369-2377. [CrossRef]

11. Trinh, K.T.L.; Trinh, T.N.D.; Lee, N.Y. Fully integrated and slidable paper-embedded plastic microdevice for point-of-care testing of multiple foodborne pathogens. Biosens. Bioelectron. 2019, 135, 120-128. [CrossRef] [PubMed]

12. Tang, W.; Chow, W.H.; Li, Y.; Kong, H.; Tang, Y.W.; Lemieux, B. Nucleic acid assay system for tier II laboratories and moderately complex clinics to detect HIV in low-resource settings. J. Infect. Dis. 2010, 201, S46. [CrossRef] [PubMed]

13. Mahalanabis, M.; Do, J.; ALMuayad, H.; Zhang, J.Y.; Klapperich, C.M. An integrated disposable device for DNA extraction and helicase dependent amplification. Biomed. Microdevices 2010, 12, 353-359. [CrossRef] [PubMed]

14. Liu, D.; Shen, H.; Zhang, Y.; Shen, D.; Zhu, M.; Song, Y.; Zhu, Z.; Yang, C. A microfluidic-integrated lateral flow recombinase polymerase amplification (MI-IF-RPA) assay for rapid COVID-19 detection. Lab Chip 2021, 21, 2019-2026. [CrossRef]

15. Shen, F.; Davydova, E.K.; Du, W.; Kreutz, J.E.; Piepenburg, O.; Ismagilov, R.F. Digital isothermal quantification of nucleic acids via simultaneous chemical initiation of recombinase polymerase amplification reactions on SlipChip. Anal. Chem. 2011, 83, 3533-3540. [CrossRef]

16. Rohrman, B.; Richards-Kortum, R. Inhibition of recombinase polymerase amplification by background DNA: A lateral flow-based method for enriching target DNA. Anal. Chem. 2015, 87, 1963-1967. [CrossRef]

17. Daher, R.K.; Stewart, G.; Boissinot, M.; Bergeron, M.G. Recombinase polymerase amplification for diagnostic applications. Clin. Chem. 2016, 62, 947-958. [CrossRef]

18. Lobato, I.M.; O'sullivan, C.K. Recombinase polymerase amplification: Basics, applications and recent advances. Trends Analyt. Chem. 2018, 98, 19-35. [CrossRef]

19. Li, J.; Macdonald, J.; von Stetten, F. Review: A comprehensive summary of a decade development of the recombinase polymerase amplification. Analyst 2019, 144, 31-67. [CrossRef]

20. Yang, B.; Kong, J.; Fang, X. Bandage-like wearable flexible microfluidic recombinase polymerase amplification sensor for the rapid visual detection of nucleic acids. Talanta 2019, 204, 685-692. [CrossRef] 
21. Kong, M.; Li, Z.; Wu, J.; Hu, J.; Sheng, Y.; Wu, D.; Lin, Y.; Li, M.; Wang, X.; Wang, S. A wearable microfluidic device for rapid detection of HIV-1 DNA using recombinase polymerase amplification. Talanta 2019, 205, 120155. [CrossRef]

22. Chang, Y.; You, H. Efficient bond of PDMS and printed circuit board with an application on continuous-flow polymerase chain reaction. BioChip J. 2020, 14, 349-357. [CrossRef]

23. Wang, Z.; Zhang, L.; Duan, S.; Jiang, H.; Shen, J.; Li, C. Kirigami-patterned highly stretchable conductors from flexible carbon nanotube-embedded polymer films. J. Mater. Chem. C 2017, 5, 8714-8722. [CrossRef]

24. Lee, T.-W.; Jeon, S.; Maria, J.; Zaumseil, J.; Hsu, J.W.P.; Rogers, J.A. Soft-contact optical lithography using transparent elastomeric stamps and application to nanopatterned organic light-emitting devices. Adv. Funct. Mater. 2005, 15, 1435-1439. [CrossRef]

25. So, J.-H.; Thelen, J.; Qusba, A.; Hayes, G.J.; Lazzi, G.; Dickey, M.D. Reversibly deformable and mechanically tunable fluidic antennas. Adv. Funct. Mater. 2009, 19,3632-3637. [CrossRef]

26. Jeong, S.H.; Zhang, S.; Hjort, K.; Hilborn, J.; Wu, Z. PDMS-based elastomer tuned soft, stretchable, and sticky for epidermal electronics. Adv. Mater. 2016, 28, 5830-5836. [CrossRef] [PubMed]

27. Tang, K.C.; Liao, E.; Ong, W.L.; Wong, J.D.S.; Agarwal, A.; Nagarajan, R.; Yobas, L. Evaluation of bonding between oxygen plasma treated polydimethyl siloxane and passivated silicon. J. Phys. Conf. Ser. 2006, 34, 155-161. [CrossRef]

28. Effenhauser, C.S.; Bruin, G.J.M.; Paulus, A.; Ehrat, M. Integrated capillary electrophoresis on flexible silicone microdevices: analysis of dna restriction fragments and detection of single DNA molecules on microchips. Anal. Chem. 1997, 69, 3451-3457. [CrossRef]

29. Khademhosseini, A.; Yeh, J.; Eng, G.; Karp, J.; Kaji, H.; Borenstein, J.; Farokhzad, O.C.; Langer, R. Cell docking inside microwells within reversibly sealed microfluidic channels for fabricating multiphenotype cell arrays. Lab Chip 2005, 12, 1380-1386. [CrossRef]

30. Kim, J.M.; Wolf, F.; Baier, S.K. Effect of varying mixing ratio of PDMS on the consistency of the soft-contact Stribeck curve for glycerol solutions. Tribol. Int. 2015, 89, 46-53. [CrossRef]

31. Trinh, K.T.L.; Zhang, H.; Kang, D.-J.; Kahng, S.-H.; Tall, B.D.; Lee, N.Y. Fabrication of polymerase chain reaction plastic lab-on-a-chip device for rapid molecular diagnoses. Int. Neurourol. J. 2016, 20, S38-S48. [CrossRef] [PubMed]

32. Brown, X.Q.; Ookawa, K.; Wong, J.Y. Evaluation of polydimethylsiloxane scaffolds with physiologically-relevant elastic moduli: Interplay of substrate mechanics and surface chemistry effects on vascular smooth muscle cell response. Biomaterials 2005, 26, 3123-3129. [CrossRef] [PubMed]

33. DeShields, J.B.; Moroz, N.; Braley, L.E.; Mora-Romero, G.A.; Tanaka, K. Recombinase polymerase amplification (RPA) for the rapid isothermal detection of Spongospora subterranean f. sp. subterranea and potato mop-top virus. Am. J. Potato Res. 2019, 96, 617-624. [CrossRef] 